\title{
REVIEW
}

\section{Is XMRV a causal virus for prostate cancer?}

\author{
Zhen-Zhen Zhang ${ }^{1,2}$, Bao-Feng Guo ${ }^{3}$, Zhuang Feng ${ }^{4}$, Ling Zhang ${ }^{2}$ and Xue-Jian Zhao ${ }^{2}$ \\ The potential association between xenotropic murine leukaemia virus-related gammaretrovirus (XMRV) and prostate cancer (PCa) has \\ been documented since 2006. It is important for furthering our understanding of the biological mechanisms of PCa to ascertain \\ whether this association is causal. To summarize the available information on the epidemiological and laboratory findings of the \\ association, we conducted a literature search of the PubMed electronic database (from March 2006 to February 2011) to identify \\ relevant published studies that examined the association between XMRV and PCa. Although several studies showed the positive \\ association between XMRV and PCa, more recent studies did not support this conclusion. The positive findings might be due to \\ contamination of human samples. Further studies are needed to clarify this association.
} Asian Journal of Andrology (2011) 13,698-701; doi:10.1038/aja.2011.32; published online 18 July 2011

Keywords: prostate cancer; review; XMRV

\section{INTRODUCTION}

Prostate cancer (PCa) is the most commonly diagnosed cancer among men in the United States (US). PCa alone accounted for 28\% (217 730 new PCa cases out of 789620 total cancer cases) of cancer incidence and 11\% (32 050 PCa deaths out of 299200 total cancer deaths) of the estimated cancer deaths among men in the US in $2010 .{ }^{1}$ On the basis of PCa cases diagnosed between 1999 and 2005, an estimated 92\% of these new cases are expected to be diagnosed at local or regional stages. ${ }^{1}$ In the US, African-American men have a relatively higher incidence of $\mathrm{PCa}$ and are twice as likely to die from PCa as Caucasian-American men. The 5-year relative survival rate is $100 \%$ for Caucasian-Americans, $98 \%$ for African-Americans and 100\% for Asian/Pacific Islanders. ${ }^{1}$

The aetiology of PCa is complex. The cancer has been associated with a range of socioeconomic, ${ }^{2}$ cultural, ${ }^{2}$ genetic and familial factors. ${ }^{3}$ A meta-analysis pooling 11 case-control studies from 1971 to 1996 reported an increased risk of PCa among men with a history of prostatitis (odds ratio $=1.57 ; 95 \%$ confidence interval: $1.01-2.45$ ). ${ }^{4}$ This meta-analysis also reported that the odds ratio increased to 1.77 (95\% confidence interval 1.05-2.98) if analysis was restricted to the six population-based case-control studies. ${ }^{4}$ The prevalence of prostatitis is in the range of $2 \%-10 \%$ among various populations in Asia, North America and Europe. ${ }^{5}$

Viral infections may cause chronic inflammation and tumourigenesis of the prostate. ${ }^{6-8}$ To date, viruses such as those of the human herpes virus family (HHP-2, HHV-8, human cytomegalovirus (HCMV or HHV-5) and Epstein-Barr virus (HHV-4)), polyomaviruses (BKV, JCV and SV40) and human papillomaviruses have been detected in association with PCa. ${ }^{9,10}$ More recently, in 2006, xenotropic murine leukaemia virus-related gammaretrovirus (XMRV), a member of the gammaretrovirus family, was isolated from human PCa tissue. ${ }^{11}$ This review will focus on summarizing the epidemiological and laboratory research progress in examining the association of XMRV and PCa, according to reports available in the PubMed electronic database (from March 2006 to February 2011).

\section{BACKGROUND OF XMRV}

XMRV was first identified to be associated with PCa by the US investigators Urisman et al. ${ }^{11}$ in their investigation of the role of viruses in the carcinogenesis of PCa in 2006. The sequence of the virus was closely related to that of the xenotropic murine leukaemia viruses (MLVs or MuLVs), and hence it was referred to as a xenotropic murine leukaemia virus-related retrovirus (XMRV). ${ }^{11}$ More specifically, it was identified as a novel gammaretrovirus. ${ }^{11}$

Mice have been hosts to retroviruses for millions of years, and there are contributions from perhaps hundreds of retroviruses in the mouse genome, with a number of phylogenetic groups identified. ${ }^{12,13}$ In contrast to MLVs, XMRV rarely infects mouse cells; indeed, it more often infects human cells, similar to other xenotropic MLVs. ${ }^{14}$ As with other retroviruses, $\mathrm{XMRV}$ is a simple RNA-containing virus that can convert RNA to DNA by reverse transcription. This DNA copy can be integrated with infected host cells' DNA and can replicate within the host. Thus, the retrovirus' genetic information can persist in the infected host cells and their descendants for an indefinite number of generations. ${ }^{14}$ Urisman et al. ${ }^{11}$ also reported that XMRV was different from other MLVs in some parts of the viral genome, such as a 24-nucleotide deletion in the gag leader, a stop codon 53 amino acid residues downstream from the alternative gag start codon, and several single-nucleotide substitutions in the long terminal repeat. However, Hue et al. ${ }^{15}$ disputed whether the 24-nucleotide deletion signature was specific to XMRV. In their studies, primers targeting the 24-nucleotide deletion were able to amplify MLV sequences in inbred and wild-derived inbred mouse strains. 


\section{GENETIC FACTORS RELATED TO THE HOST SUSCEPTIBILITY TO XMRV}

XMRV infection of human tissue cells also depends on the specific host's genetic characteristics that affect the immune response. One of the important genetic mutations involves the RNASEL gene, which encodes the protein ribonuclease L (RNase L), an endoribonuclease that is essential for anti-viral defence of the host. ${ }^{11} \mathrm{R} 462 \mathrm{Q}$ polymorphisms in the RNASEL gene have been reported to result in a reducedactivity variant of the innate anti-viral factor RNase L. ${ }^{11}$ RNASEL plays a role in anti-viral activity mediated through interferon. ${ }^{16,17}$ Although it was initially proposed that the homozygous QQ genotype of RNASEL R462Q was associated with XMRV, ${ }^{11}$ these results were not reproduced in later studies. ${ }^{18,19}$

Groom et l. $^{20}$ found that two of the three major classes of retroviral restriction factors identified to date were associated with restricting XMRV replication. These two factors include apolipoprotein B mRNA editing complex 3 (APOBEC3) and tetherin proteins, which may act to inhibit viral DNA synthesis ${ }^{21-23}$ and restrict the release of newly generated retroviral particles, ${ }^{24}$ respectively. ${ }^{20}$ Paprotka et al. ${ }^{25}$ found that XMRV preferentially infected cells such as PCa cells expressing low or nearly undetectable mRNA levels of APOBEC3F and APOBEC3G.

\section{METHODS FOR DETECTING XMRV IN PCA IN LABORATORIES}

Several laboratory methods can be used to detect XMRV, e.g., polymerase chain reaction (PCR), reverse transcriptase-polymerase chain reaction, immunohistochemistry, fluorescence in situ hybridisation, ${ }^{14,26}$ serum assay ${ }^{27}$ and virus neutralisation and culture. ${ }^{28}$ DNA sequence analysis has been used to detect the RNASEL G1385A (R462Q) mutation. ${ }^{19,29}$ Sensitivity analysis of the PCR assay can be performed using a PCR calibrator. ${ }^{29}$ Danielson et al. ${ }^{19}$ suggested that the detection of XMRV required specific PCR conditions. Their study showed that env primers were a more sensitive choice for XMRV detection than gag and pol primers; they also recommended using at least $600 \mathrm{ng}\left(1 \times 10^{5}\right.$ cells $)$ prostatic tissue DNA for reliable PCR detection of XMRV. However, Aloia et al. ${ }^{14}$ suggested that the extraordinary sensitivity of the PCR technique increased the risk of finding false positives. More recently, Sakakibara et al. ${ }^{30}$ reported that factors that could lead to NF- $\mathrm{BB}$ activation would enhance XMRV transcription and replication.

\section{EPIDEMIOLOGICAL STUDIES IN HUMANS}

XMRV was first discovered in 2006 by Urisman et al. ${ }^{11}$ at the University of California, San Francisco. Among $11 \mathrm{PCa}$ patients with the homozygous R462Q (QQ) genotype, seven were found to be positive for XMRV, as detected by DNA microarray analysis. Further detection of 20 R462Q (QQ) cases by reverse transcriptase-polymerase chain reaction found that $40 \%$ of them were positive for XMRV. ${ }^{11}$ A case-control study of $334 \mathrm{PCa}$ tissues (233 cases vs. 101 benign controls) at Columbia University Medical Center demonstrated that XMRV protein was detected in $23 \%$ of PCa cases and $4 \%$ of controls, independent of RNASEL gene mutation. ${ }^{18}$ The study also found that the higher the grade of tumour (as measured by the Gleason score), the higher the prevalence of XMRV protein positivity ( $P=0.06$ for trend analysis). ${ }^{18}$ Another study using a novel serum assay at Emory University found serological evidence of XMRV in 11 out of 40 patients (27.5\%). The prevalence of XMRV was higher among those patients with the RNASEL R462Q (QQ) genotype than among those with other genotypes $(40 \%$ vs. $15 \%) .{ }^{27}$ A retrospective study in the southern US (Baylor College of Medicine), which enrolled 114 postprostatectomy $\mathrm{PCa}$ patients with a family history of $\mathrm{PCa}$ in at least one first- or second-degree relative, demonstrated that XMRV was present in 22\% (32 out of 144) of PCa patients; however, XMRV infection did not correlate with RNASEL R462Q genotype. ${ }^{19}$ The proportion of XMRV positivity in this southern US study was similar to that obtained in the previous two studies of PCa patients in the US. ${ }^{18,27}$

In contrast, several studies in the US reported the absence, or extremely low prevalence, of XMRV in PCa. For instance, Sfanos et al. ${ }^{31}$ did not detect any XMRV DNA in 338 samples representing tumour, normal and benign prostate hyperplasia (BPH) tissues from 200 PCa patients. In contrast to the previous report that the virus was most frequent in patients homozygous for the R462Q variant QQ, ${ }^{11}$ PCa patients in this study were less likely to have the RNASEL QQ variant. ${ }^{31}$ Another research study conducted by Aloia et al. ${ }^{14}$ did not find any signs of XMRV infection using two assays (real-time PCR and immunohistochemistry with two rabbit polyclonal antisera) and surveying nearly 800 prostate tumours prepared as full tumour tissue sections or tumour tissue microarrays, including microdissected prostate tumour, metastatic prostate tumour and intermediate as well as high-grade primary prostate tumour tissues.

Investigators conducting studies on the associations between $\mathrm{XMRV}$ and $\mathrm{PCa}$ in Europe have reported either rare detection of XMRV or negative findings. One case-control study by Fischer et al. ${ }^{32}$ reported that $0.95 \%$ ( 1 out of 105 ) of PCa patients were positive for XMRV compared to $1.42 \%$ ( 1 out of 70 ) of healthy controls. In addition, the prevalences of the homozygous mutation R462Q (QQ), as determined by RNASEL genotyping of SNP R462Q, were $8.04 \%$ for PCa cases and $5.71 \%$ for controls. ${ }^{32}$ Two years later, the same research group reported finding XMRV sequences in respiratory tract secretions among 329 immunocompetent and immunocompromised patients, indicating the possible transmission of XMRV through the respiratory system. ${ }^{33}$ Another study examined $500 \mathrm{PCa}$ tissue samples and did not find evidence of XMRV infection. ${ }^{34}$ One study reported that $4 \%$ (3 out of 74 ) of PCa patients were positive for XMRV in their prostatic tissue, and two of the three XMRV-positive patients displayed a homozygous RNASEL G1385A genotype (G/G). ${ }^{29}$ This result may be due to either a low prevalence of XMRV in the area where the study was performed or the unnatural presence of XMRV in seminal plasma, as suggested by a study investigating the presence of XMRV in seminal plasma samples from HIV-1-infected men in this population. ${ }^{35}$ One study did not find any evidence of XMRV infection in the tissue samples of nine PCa patients selected from 139 patients after genotyping for R462Q mutations. ${ }^{36}$

In addition, in contrast to the original finding by Urisman et al., ${ }^{11} \mathrm{a}$ case-control study on Mexican PCa patients failed to find any XMRV infection among $52 \mathrm{PCa}$ cases and found only one XMRV-positive sample, with an R462 R/R genotype, out of 75 control samples. ${ }^{9}$ The prevalence of XMRV among these subjects was much lower than that reported in the study by Urisman et al. ${ }^{11}$

\section{XMRV-RELATED FINDINGS AND CLINICAL CHARACTERISTICS OF PCA}

\section{Cell types}

In terms of cell types infected by XMRV in the prostate, the original study discovered the presence of infection exclusively in non-malignant stromal and haematopoietic cells adjacent to carcinoma. ${ }^{11}$ In contrast, another study found that XMRV was primarily located in prostate carcinoma cells. ${ }^{18} \mathrm{~A}$ more recent study in the southern US 
reported that XMRV was detectable in both normal and tumour prostate tissues from PCa patients, independent of R462Q genotype, which suggested that XMRV infection might precede cancer onset. ${ }^{19}$ Therefore, the role of XMRV infection in prostate carcinogenesis remains to be clarified. ${ }^{10}$

\section{Clinical pathological parameters}

To date, current studies reporting the association of XMRV and various clinical pathological parameters of PCa are limited. Only two studies report the correlation of XMRV with Gleason score: Schlaberg et al. ${ }^{18}$ found a correlation of XMRV infection with high Gleason scores; Danielson et al. ${ }^{19}$ also found a slight trend in favour of an increasing Gleason score, but the result was not statistically significant. Additional studies will need to include larger numbers of patients to evaluate the correlation between XMRV infection and clinical pathological parameters, including Gleason scores, seminal vesicle invasion, extracapsular extension, surgical margin invasion and lymph node involvement. These parameters are important factors to determine the stage of $\mathrm{PCa},{ }^{37}$ which is critical information for clinicians who wish to prescribe appropriate treatments and enhance survival rate. $^{38}$

\section{XMRV ASSOCIATED WITH PCA AND CHRONIC FATIGUE SYNDROME}

In addition to PCa, XMRV has been associated with a second human disease: chronic fatigue syndrome (CFS). Impaired regulation of the 25A synthetase/RNase L anti-viral pathway was reported to be associated with individuals with CFS. ${ }^{39}$ In 2009, Lombardi et al. ${ }^{28}$ found 68 XMRV-positive subjects out of 101 patients (67\%) with severe CFS compared to 8 out of 218 healthy controls (3.7\%), suggesting a causal role for XMRV in the pathogenesis of CFS. These two human disorders (PCa and CFS) are the only two human diseases that have been found to be associated with XMRV. At present, studies have detected XMRV at widely different rates in patients with $\mathrm{PCa}(0 \%-27 \%)$ and in patients with CFS $(0 \%-67 \%){ }^{26}$ Two studies conducted in the United Kingdom after 2009 failed to show evidence of association between XMRV and CFS. ${ }^{40,41}$ A matched case-control study also failed to demonstrate the presence of XMRV in mononuclear cells from peripheral blood of CFS patients. ${ }^{42}$ These three studies all cast doubt on the association between XMRV and CFS, similar to the findings for PCa. Furthermore, a cross-sectional study did not find any positive XMRV cases among enrolled patients with CFS, with other immunomodulatory diseases, such as HIV infection or rheumatoid arthritis, with haematopoietic stem-cell transplantation or solid organ transplantation, or among general patients seeking medical care. ${ }^{43}$ To date, there are no laboratory data demonstrating the effects of XMRV on PCa and CFS.

\section{CONTAMINATION IS A POTENTIAL PROBLEM}

The factors responsible for the broad, inconsistent associations between XMRV prevalence and PCa/CFS are not well understood. However, there are concerns that human samples might have been contaminated with mouse DNA. Smith ${ }^{44}$ reviewed four recent articles about mouse DNA contamination in XMRV-related research ${ }^{15,45-47}$ and summarized three potential sources of contamination in studies of XMRV utilizing PCR-based techniques. The author reported that MLV-encoding nucleic acids have been detected in commercial PCR reagents and that mouse genomic DNA has been detected in human blood and tissue samples. In addition, nucleic acids of human tumour cell lines have been infected by XMRV or other gammaretroviruses. ${ }^{44}$

\section{CONCLUSIONS}

The role of infectious agents and chronic inflammation in carcinogenesis is increasingly being recognized. Given that viruses have been found in some cancerous tissues from PCa patients, the possibility that exposure to viral infection may contribute to PCa incidence has been considered. Since 2006, the role of XMRV in human disease has been intensively investigated, but its prevalence, geographical distribution and disease associations are still not clear. ${ }^{48}$ The patients included in all of the past studies were not randomly selected, and both internal and external validity issues need to be investigated. There is no convincing evidence that exposure to XMRV plays a causal role in the pathogenesis of PCa. Many factors, including geographical location, study design, detection approaches in the laboratory, patient selection, analytical methods and control of confounding factors, have contributed to the inconsistent detection of XMRV among different human populations. ${ }^{48}$ In addition, it is suggested that potential contamination of human samples with mouse DNA can lead to the false positive detection of XMRV. ${ }^{15,44-47}$

More research is needed to overcome the deficiencies in the data available to date. First, standardized and generally accepted assays for XMRV need to be developed. More sensitive assays for XMRV detection and isolation of infectious XMRV from PCa patients are also warranted. Second, large-scale epidemiological examination of the incidence and prevalence of XMRV among PCa patients may further help to explain the potential association. Given the higher incidence and lower survival rates of African-Americans with PCa compared to Caucasian-Americans and Asians, specific ethnic groups especially African-Americans should be targeted in future research to determine whether there are any racial differences in the association between XMRV infection and PCa. The investigation and resolution of these issues will improve our understanding of PCa and facilitate its prevention, diagnosis and treatment.

\section{COMPETING FINANCIAL INTERESTS}

The authors declare no competing financial interests.

\section{ACKNOWLEDGMENTS}

This work was funded by the National Natural Science Foundation of China (No. 30801354 and No. 30970791) and Jilin Provincial Science and Technology Department, China (grant No. 20080154).

1 Jemal A, Siegel R, Xu J, Ward E. Cancer statistics, 2010. CA Cancer J Clin 2010; 60: 277-300.

2 Raymundo EM, Rice KR, Chen Y, Zhao J, Brassell SA. Prostate cancer in Asian Americans: incidence, management and outcomes in an equal access healthcare system. BJU Int 2011; 107: 1216-22.

3 Stein QP, Flanagan JD. Genetic and familial factors influencing breast, colon, prostate and lung cancers. S D Med 2010; Spec No.: 16-22.

4 Dennis LK, Lynch CF, Torner JC. Epidemiologic association between prostatitis and prostate cancer. Urology 2002; 60: 78-83.

$5 \mathrm{Ku} \mathrm{JH}, \mathrm{Kim}$ SW, Paick JS. Epidemiologic risk factors for chronic prostatitis. Int J Androl 2005; 28: 317-27.

6 de Marzo AM, Platz EA, Sutcliffe S, Xu J, Gronberg H et al. Inflammation in prostate carcinogenesis. Nat Rev Cancer 2007; 7: 256-69.

7 Klein EA, Silverman R. Inflammation, infection, and prostate cancer. Curr Opin Urol 2008; 18: 315-9.

8 Nelson WG, de Marzo AM, DeWeese TL, Isaacs WB. The role of inflammation in the pathogenesis of prostate cancer. J Urol 2004; 172: S6-11.

9 Martinez-Fierro ML, Leach RJ, Gomez-Guerra LS, Garza-Guajardo R, Johnson-Pais T et al. Identification of viral infections in the prostate and evaluation of their association with cancer. BMC Cancer 2010; 10: 326.

10 Rajarubendra N, Lawrentschuk N, Bolton DM, Klotz L, Davis ID. Prostate cancer immunology—an update for Urologists. BJU Int 2011; 107: 1046-51. 
11 Urisman A, Molinaro RJ, Fischer N, Plummer SJ, Casey G et al. Identification of a nove Gammaretrovirus in prostate tumors of patients homozygous for R462Q RNASEL variant. PLoS Pathog 2006; 2: e25.

12 Coffin JM, Stoye JP, Frankel WN. Genetics of endogenous murine leukemia viruses. Ann N Y Acad Sci 1989; 567: 39-49.

13 Stocking C, Kozak CA. Murine endogenous retroviruses. Cell Mol Life Sci 2008; 65 3383-98.

14 Aloia AL, Sfanos KS, Isaacs WB, Zheng Q, Maldarelli F et al. XMRV: a new virus in prostate cancer? Cancer Res 2010; 70: 10028-33.

15 Hue S, Gray ER, Gall A, Katzourakis A, Tan CP et al. Disease-associated XMRV sequences are consistent with laboratory contamination. Retrovirology 2010; 7: 111 .

16 Silverman $\mathrm{RH}$. A scientific journey through the 2-5A/RNase L system. Cytokine Growth Factor Rev 2007; 18: 381-8.

17 Silverman $\mathrm{RH}$. Viral encounters with 2',5'-oligoadenylate synthetase and RNase L during the interferon antiviral response. J Virol 2007; 81: 12720-9.

18 Schlaberg R, Choe DJ, Brown KR, Thaker HM, Singh IR. XMRV is present in malignan prostatic epithelium and is associated with prostate cancer, especially high-grade tumors. Proc Natl Acad Sci USA 2009; 106: 16351-6.

19 Danielson BP, Ayala GE, Kimata JT. Detection of xenotropic murine leukemia virusrelated virus in normal and tumor tissue of patients from the southern United States with prostate cancer is dependent on specific polymerase chain reaction conditions. J Infect Dis 2010; 202: 1470-7.

20 Groom HC, Yap MW, Galao RP, Neil SJ, Bishop KN. Susceptibility of xenotropic murine leukemia virus-related virus (XMRV) to retroviral restriction factors. Proc Natl Acad Sci U S A 2010; 107: 5166-71.

21 Malim MH. APOBEC proteins and intrinsic resistance to HIV-1 infection. Philos Trans $R$ Soc Lond B Biol Sci 2009; 364: 675-87.

22 Sheehy AM, Gaddis NC, Choi JD, Malim MH. Isolation of a human gene that inhibits HIV-1 infection and is suppressed by the viral Vif protein. Nature2002; 418: 646-50.

23 Holmes RK, Malim MH, Bishop KN. APOBEC-mediated viral restriction: not simply editing? Trends Biochem Sci 2007; 32: 118-28.

24 Neil SJ, Zang T, Bieniasz PD. Tetherin inhibits retrovirus release and is antagonized by HIV-1 Vpu. Nature 2008; 451: 425-30.

25 Paprotka T, Venkatachari NJ, Chaipan C, Burdick R, Delviks-Frankenberry KA et al. Inhibition of xenotropic murine leukemia virus-related virus by APOBEC3 proteins and antiviral drugs. J Virol 2010; 84: 5719-29.

26 Silverman RH, Nguyen C, Weight CJ, Klein EA. The human retrovirus XMRV in prostate cancer and chronic fatigue syndrome. Nat Rev Urol 2010; 7: 392-402.

27 Arnold RS, Makarova NV, Osunkoya AO, Suppiah S, Scott TA et al. XMRV infection in patients with prostate cancer: novel serologic assay and correlation with PCR and FISH. Urology 2010; 75: 755-61.

28 Lombardi VC, Ruscetti FW, das Gupta J, Pfost MA, Hagen KS et al. Detection of an infectious retrovirus, XMRV, in blood cells of patients with chronic fatigue syndrome. Science 2009; 326: 585-9.

29 Verhaegh GW, de Jong AS, Smit FP, Jannink SA, Melchers WJ et al. Prevalence of human xenotropic murine leukemia virus-related gammaretrovirus (XMRV) in dutch prostate cancer patients. Prostate 2011; 71: 415-20.

30 Sakakibara S, Sakakibara K, Tosato G. NF-kappaB activation stimulates transcription and replication of xenotropic murine leukemia virus-related virus in human B-lineage and prostate carcinoma cells. J Virol 2011; 85: 3179-86.
31 Sfanos KS, Sauvageot J, Fedor HL, Dick JD, de Marzo AM et al. A molecular analysis of prokaryotic and viral DNA sequences in prostate tissue from patients with prostate cancer indicates the presence of multiple and diverse microorganisms. Prostate 2008; 68: 306-20.

32 Fischer N, Hellwinkel O, Schulz C, Chun FK, Huland $\mathrm{H}$ et al. Prevalence of human gammaretrovirus XMRV in sporadic prostate cancer. J Clin Virol 2008; 43: 277-83.

33 Fischer N, Schulz C, Stieler K, Hohn O, Lange C et al. Xenotropic murine leukemia virus-related gammaretrovirus in respiratory tract. Emerg Infect Dis 2010; 16 1000-2.

34 Hohn O, Krause H, Barbarotto P, Niederstadt L, Beimforde N et al. Lack of evidence for xenotropic murine leukemia virus-related virus (XMRV) in German prostate cancer patients. Retrovirology 2009; 6: 92.

35 Cornelissen M, Zorgdrager F, Blom P, Jurriaans S, Repping S et al. Lack of detection of XMRV in seminal plasma from HIV-1 infected men in The Netherlands. PLoS One 2010; 5: e12040.

36 D'Arcy F, Foley R, Perry A, Marignol L, Lawler M et al. No evidence of XMRV in Irish prostate cancer patients with the R462Q mutation. Eur Urol Supp/ 2009; 7: 271.

37 Hoedemaeker RF, Vis AN, van der Kwast TH. Staging prostate cancer. Microsc Res Tech 2000; 51: 423-9.

38 Scherr D, Swindle PW, Scardino PT. National Comprehensive Cancer Network guidelines for the management of prostate cancer. Urology 2003; 61: 14-24.

39 Suhadolnik RJ, Reichenbach NL, Hitzges P, Sobol RW, Peterson DL et al. Upregulation of the 2-5A synthetase/RNase $L$ antiviral pathway associated with chronic fatigue syndrome. Clin Infect Dis 1994; 18 (Suppl 1): S96-104.

40 Erlwein O, Kaye S, McClure MO, Weber J, Wills G et al. Failure to detect the nove retrovirus XMRV in chronic fatigue syndrome. PLoS One 2010; 5: e8519.

41 Groom HC, Boucherit VC, Makinson K, Randal E, Baptista S et al. Absence of xenotropic murine leukaemia virus-related virus in UK patients with chronic fatigue syndrome. Retrovirology 2010; 7: 10.

42 van Kuppeveld FJ, de Jong AS, Lanke KH, Verhaegh GW, Melchers WJ et al. Prevalence of xenotropic murine leukaemia virus-related virus in patients with chronic fatigue syndrome in the Netherlands: retrospective analysis of samples from an established cohort. BMJ 2010; 340: c1018.

43 Henrich TJ, Li JZ, Felsenstein D, Kotton CN, Plenge RM et al. Xenotropic murine leukemia virus-related virus prevalence in patients with chronic fatigue syndrome or chronic immunomodulatory conditions. J Infect Dis 2010; 202: 1478-81.

44 Smith RA. Contamination of clinical specimens with MLV-encoding nucleic acids: implications for XMRV and other candidate human retroviruses. Retrovirology 2010; 7: 112

45 Oakes B, Tai AK, Cingoz O, Henefield MH, Levine S et al. Contamination of human DNA samples with mouse DNA can lead to false detection of XMRV-like sequences. Retrovirology 2010; 7: 109.

46 Robinson MJ, Erlwein OW, Kaye S, Weber J, Cingoz 0 et al. Mouse DNA contamination in human tissue tested for XMRV. Retrovirology 2010; 7: 108.

47 Sato E, Furuta RA, Miyazawa T. An endogenous murine leukemia viral genome contaminant in a commercial RT-PCR Kit is amplified using standard primers for XMRV. Retrovirology 2010; 7: 110.

48 Kearney M, Maldarelli F. Current status of xenotropic murine leukemia virus-related retrovirus in chronic fatigue syndrome and prostate cancer: reach for a scorecard, not a prescription pad. J Infect Dis 2010; 202: 1463-6. 